\title{
Closed-loop electrophysiological experiments and metadata management with RELACS and LabLog Jan Benda* and Jan Grewe
}

\author{
Address: Department Biology II, Ludwig-Maximilian University, 82152 Martinsried, Germany \\ Email: Jan Benda* - benda@bio.lmu.de \\ * Corresponding author
}

from Eighteenth Annual Computational Neuroscience Meeting: CNS*2009

Berlin, Germany. 18-23 July 2009

Published: 29 September 2009

BMC Neuroscience 2009, I0(Suppl I):S2 doi: I0.I I86/I47I-2202-I0-SI-S2

This abstract is available from: http://www.biomedcentral.com/I47I-2202/I0/SI/S2

(c) 2009 Benda and Grewe; licensee BioMed Central Ltd.

For many electrophysiological experiments, time is precious. Online analysis and visualization, automated experimental protocols, and closed-loop experimental designs that generate stimuli based on the recorded responses considerably improve the yield of a recording. For example, simply displaying processed results online in addition to the raw voltage traces already gives the experimenter valuable quantitative information during a running experiment. Probing the neuron with stimuli that are outside its dynamic range can be avoided. Advancing individual electrodes can be automated, increasing the chances of a dual recording.

In addition, closed-loop experiments are the basis for novel experimental designs [1]. For example, stimulus parameters can be tuned iteratively to find the optimal stimulus that maximizes the mutual information between the stimulus and the evoked spike-train response. Also, finding sets of stimulus parameter that result in the same response can be implemented much more efficiently on a closed-loop system. This iso-response method is a powerful method for characterizing neuronal filter properties.

RELACS [2] is a fully customizable software platform specifically designed for closed-loop electrophysiological experiments. Filters and spike detectors can be applied instantly on the recorded potentials. Freely programmable, hardware-independent $\mathrm{C}++$ plugins can access the preprocessed data for further online analysis, visualization, and stimulus generation.
Data can be reused much more easily if they are annotated with metadata that specify the conditions under which the dataset has been recorded, describe the applied stimuli and provide general information about the recorded cell and the experimental setup, as well as an outline of the experimental objectives. RELACS automatically stores most of such metadata during each recording.

The metadata are stored in a MySQL database that is managed by LabLog [3]. This way, the recorded metadata are immediately secured for long-term data management and data mining purposes. Early and automatic handling of the data and their metadata is one of the key issues for data sharing with colleagues or dealing with public data repositories. Since metadata play a fundamental role for data management, data sharing and data analysis, an interface definition is needed. We therefore propose a simple XML schema that specifies metadata by properties (extended key-value pairs) that are organized in a hierarchical structure. Within this schema, we suggest a common and extensible vocabulary for key properties to make the metadata understandable across applications.

\section{References}

I. Benda J, Gollisch T, Machens CK, Herz AVM: From response to stimulus: adaptive sampling in sensory physiology. Curr Opin Neurobiol 2007, 17:430-436.

2. RELACS - Relaxed Electrophysiological Data Acquisition, Control, and Stimulation [http://www.relacs.net]

3. LabLog - Laboratory Logbook [http://lablog.sourceforge.net] 